\title{
SIGN CHANGING SOLUTIONS WITH CLUSTERED LAYERS NEAR THE ORIGIN FOR SINGULARLY PERTURBED SEMILINEAR ELLIPTIC PROBLEMS ON A BALL*
}

\author{
YIHONG DU ${ }^{\dagger}$, ZHAOLI LIU ${ }^{\ddagger}$, ANGELA PISTOIA ${ }^{\S}$, AND SHUSEN YAN $₫$ \\ Dedicated to Professor Neil Trudinger on the occasion of his 65th birthday
}

Abstract. We study sign changing solutions to equations of the form

$$
-\epsilon^{2} \Delta u+u=f(u) \text { in } B, \partial_{\nu} u=0 \text { on } \partial B,
$$

where $B$ is the unit ball in $\mathbb{R}^{N}(N \geq 2), \epsilon$ is a positive constant and $f(u)$ behaves like $|u|^{p-1} u$ (but not necessarily odd) with $1<p<(N+2) /(N-2)$ if $N \geq 3$, and $1<p<\infty$ if $N=2$. We show that for any given positive integer $n$, this problem has a sign changing radial solution $v_{\epsilon}(|x|)$ which changes sign at exactly $n$ spheres $\cup_{j=1}^{n}\left\{|x|=\rho_{j}^{\epsilon}\right\}$, where $0<\rho_{1}^{\epsilon}<\cdots<\rho_{n}^{\epsilon}<1$ and as $\epsilon \rightarrow 0$, $\rho_{j}^{\epsilon} \rightarrow 0$ and $v_{\epsilon}(r) \rightarrow 0$ uniformly on compact subsets of $(0,1]$. Moreover, given any sequence $\epsilon_{k} \rightarrow 0$, there is a subsequence $\epsilon_{k_{i}}$ such that $u_{\epsilon}(|x|):=v_{\epsilon}(\epsilon|x|)$ converges to some $U$ in $C_{l o c}^{1}\left(\mathbb{R}^{N}\right)$ along this subsequence, and $U=U(|x|)$ is a radial sign changing solution of

$$
-\Delta U+U=f(U) \text { in } \mathbb{R}^{N}, U \in H^{1}\left(\mathbb{R}^{N}\right)
$$

with exactly $n$ zeros: $0<\rho_{1}<\cdots<\rho_{n}<\infty$, and $\epsilon^{-1} \rho_{j}^{\epsilon} \rightarrow \rho_{j}$ along the subsequence $\epsilon_{k_{i}}$. Hence the sharp layers of the sign changing solution $v_{\epsilon}$ are clustered near the origin.

The same result holds if the Neumann boundary condition is replaced by the Dirichlet boundary condition, or if $B$ is replaced by $\mathbb{R}^{N}$.

Key words. Clustered layers, singular perturbation, semilinear elliptic equation.

AMS subject classifications. Primary 35B45; Secondary 35J40

1. Introduction. Positive solutions of the singularly perturbed Neumann problem

$$
-\epsilon^{2} \Delta u+u=f(u) \text { in } \Omega, \partial_{\nu} u=0 \text { on } \partial \Omega
$$

have been investigated extensively in the past decade. Here $\Omega$ is a bounded smooth domain in $\mathbb{R}^{N}(N \geq 2), \epsilon$ is a positive constant and $f(u)$ behaves like $u^{p}$ with $1<$ $p<(N+2) /(N-2)$ if $N \geq 3$, and $1<p<\infty$ if $N=2$. It is known that for small $\epsilon$, (1.1) has positive solutions with sharp peaks concentrating at certain interior points as well as on the boundary of $\Omega$; for example, it was shown in [GW] that given any nonnegative integers $k$ and $n$ with $k+n>0$, (1.1) has, for small $\epsilon>0$, a positive solution which concentrates at exactly $k$ peaks in the interior of $\Omega$ and $n$ peaks on the boundary of $\Omega$. For more results and background, we refer to the survey $[\mathrm{N}]$. Positive

*Received February 27, 2008; accepted for publication July 31, 2008. Research partially supported by the Australian Research Council, NSF of China (10771117), NSF of China (10571123) and Mi.U.R. Project "Metodi variazionali e topologici nello studio di fenomeni non lineari".

${ }^{\dagger}$ Department of Mathematics, School of Science and Technology, University of New England, Armidale, NSW 2351, Australia (ydu@mcs.une.edu.au); Department of Mathematics, Qufu Normal University, Qufu, Shandong 273165, P.R. China.

${ }^{\ddagger}$ Department of Mathematics, Capital Normal University, Beijing 100037, P.R. China (zliu@mail. cnu.edu.cn).

${ }^{\S}$ Dipartimento di Metodi e Modelli Mathematici, Univ. di Roma "la Sapienza", via A. Scarpa 16, 00161 Roma, Italy (pistoia@dmmm.uniroma1.it).

IDepartment of Mathematics, School of Science and Technology, University of New England, Armidale, NSW 2351, Australia (syan@mcs.une.edu.au). 
solutions with sharp layers also exist; see for example [MN], [AMN1], [AMN2] and the references therein. Recently, for the case that $\Omega$ is the unit ball, it was shown in [MNW] that given any positive integer $n$, for all small $\epsilon,(1.1)$ has a positive radial solution $u_{\epsilon}$ which concentrates at $n$ spheres $\cup_{j=1}^{n}\left\{|x|=r_{j}^{\epsilon}\right\}$, where $1>r_{1}^{\epsilon}>\cdots>r_{n}^{\epsilon}$ are such that $r_{j-1}^{\epsilon}-r_{j}^{\epsilon} \sim \epsilon \log \frac{1}{\epsilon}, j=1, \ldots, N, r_{0}^{\epsilon}=1$. Thus the sharp layers are clustered at the boundary of $\Omega$.

Much less is known for sign changing solutions of (1.1). In this paper, we show that if $\Omega$ is the unit ball as in [MNW], and if $f(u)$ behaves like $|u|^{p-1} u$ (with $p$ as above), but not necessarily odd in $u$, then for any given positive integer $n$, problem (1.1) has a sign changing radial solution $v_{\epsilon}(|x|)$ which changes sign at exactly $n$ spheres $\cup_{j=1}^{n}\left\{|x|=\rho_{j}^{\epsilon}\right\}$, where $0<\rho_{1}^{\epsilon}<\cdots<\rho_{n}^{\epsilon}<1$ and as $\epsilon \rightarrow 0, \rho_{j}^{\epsilon} \rightarrow 0$ and $v_{\epsilon}(r) \rightarrow 0$ uniformly on compact subsets of $(0,1]$. Moreover, given any sequence $\epsilon_{k} \rightarrow 0$, there is a subsequence $\epsilon_{k_{i}}$ such that $u_{\epsilon}(|x|):=v_{\epsilon}(\epsilon|x|)$ converges to some $U$ in $C_{l o c}^{1}\left(\mathbb{R}^{N}\right)$ along this subsequence, and $U=U(|x|)$ is a radial sign changing solution of

$$
-\Delta U+U=f(U) \text { in } \mathbb{R}^{N}, U \in H^{1}\left(\mathbb{R}^{N}\right),
$$

$U(r)$ has exactly $n$ zeros: $0<\rho_{1}<\cdots<\rho_{n}<\infty$, and $\epsilon^{-1} \rho_{j}^{\epsilon} \rightarrow \rho_{j}$ along the subsequence $\epsilon_{k_{i}}$. Hence in contrast to the positive solution $u_{\epsilon}$ of [MNW], the sharp layers of the sign changing solution $v_{\epsilon}$ are clustered near the origin, spaced apart in the order of $\epsilon$, but in general not evenly in the limit.

Sign changing solutions for singularly perturbed problems similar to (1.1) over a bounded domain with Dirichlet boundary conditions, or over $\mathbb{R}^{N}$, have been studied in several papers; see for example [CDNY], $[\mathrm{BCW}],[\mathrm{DP}]$ and the references therein. However, the solutions obtained in these papers are peaked solutions, namely they concentrate at isolated points, and the profile of the peak is determined by a positive solution of the corresponding entire space problem. Moreover, the approach in [CDNY] requires a special geometric condition on the underlying domain (which is not satisfied by a ball). The approaches in the other papers rely on an $x$-dependent term in the equation whose behavior determines the existence of such solutions and the location of the peaks; for a homogeneous problem like (1.1) these approaches do not work in general. In contrast, our analysis does not rely on such an $x$-dependent term, and the asymptotic profile of our solution is determined by a sign changing entire space solution. To our knowledge, this seems the first research on solutions of singularly perturbed elliptic problems with sharp layers clustered near a point.

The proof of our result is relatively simple, and relies on the radial symmetry of the problem. We follow a well-known approach which uses the Nehari manifold and a minimization argument over functions obtained by piecing together a prescribed number of positive and negative solutions of (1.1) over neighboring annuli, and estimates of the energy levels of each such positive and negative solutions. Such an approach has been developed and refined in [CSS], [CZ], [BW], [LW] and many other papers. In this paper, we mainly follow $[\mathrm{BW}]$.

This approach can treat equations more general than (1.1). More precisely, we consider the following problem

$$
-\epsilon^{2} \Delta u+b(|x|) u=f(|x|, u) \text { in } B, \partial_{\nu} u=0 \text { on } \partial B,
$$

where $B$ is the unit ball in $R^{N}(N \geq 2), b:[0,1] \rightarrow \mathbb{R}^{+}, f:[0,1] \times \mathbb{R}^{1} \rightarrow \mathbb{R}^{1}$ are continuous functions, and $f$ is locally Lipschitz in $u$ uniformly for $r \in[0,1]$. Moreover, as in $[\mathrm{BW}]$, we make the following assumptions: 
(B) $b(r) \geq a_{0}>0$ for all $r \in[0,1]$.

(F1) There exist $a_{1}, R>0$ such that

$$
|f(r, u)| \leq a_{1}|u|^{s} \text { for } r \in[0,1],|u| \geq R,
$$

where $1<s<(N+2) /(N-2)$ if $N \geq 3,1<s<\infty$ if $N=2$.

(F2) There exists $\mu>2$ such that

$$
\mu F(r, u):=\mu \int_{0}^{u} f(r, v) d x \leq u f(r, u) \text { for } r \in[0,1], u \in \mathbb{R}^{1} .
$$

(F3) $\lim _{K \rightarrow \infty} \inf _{r \in[0,1],|u| \geq K} F(r, u)>0$.

(F4) $\lim _{|u| \rightarrow 0} f(r, u) / u=0$ uniformly in $r \in[0,1]$.

(F5) $f(r, u) / u$ is increasing in $u \in \mathbb{R}^{1} \backslash\{0\}$ for every $r \in[0,1]$.

Our main result in this paper is the following theorem.

TheOREm 1.1. Suppose that (B) and (F1)-(F5) are satisfied. Then for every integer $k \geq 1$, there exists a pair of radial solutions $u_{\epsilon}^{+}(|x|)$ and $u_{\epsilon}^{-}(|x|)$ of (1.3), with $u_{\epsilon}^{-}(0)<0<u_{\epsilon}^{+}(0)$, having precisely $k$ zeros: $0<\rho_{1, \epsilon}^{ \pm}<\cdots<\rho_{k, \epsilon}^{ \pm}<1$. Moreover, as $\epsilon \rightarrow 0, \rho_{j, \epsilon}^{ \pm} \rightarrow 0$ for $j=1, \ldots, k$ and $u_{\epsilon}^{ \pm}(r) \rightarrow 0$ uniformly on compact subsets of $(0,1]$, and for any positive sequence $\epsilon_{n} \rightarrow 0$, there exists a subsequence $\epsilon_{n_{i}}$ such that $U_{\epsilon}^{ \pm}(|x|):=u_{\epsilon}^{ \pm}(\epsilon|x|)$ converges to some $U^{ \pm}$in $C_{\text {loc }}^{1}\left(\mathbb{R}^{N}\right)$ along this subsequence, and $U^{ \pm}(|x|)$ is a pair of radial sign changing solutions of

$$
-\Delta U+b(0) U=f(0, U) \text { in } \mathbb{R}^{N}, U \in H^{1}\left(\mathbb{R}^{N}\right)
$$

satisfying $U^{-}(0)<0<U^{+}(0)$, with exactly $k$ zeros: $0<\rho_{1}^{ \pm}<\cdots<\rho_{k}^{ \pm}<\infty$, and $\epsilon^{-1} \rho_{j, \epsilon}^{ \pm} \rightarrow \rho_{j}^{ \pm}$along the subsequence $\epsilon_{n_{i}}$.

REMARK 1.2. The conditions (F2), (F3) and (F5) can be relaxed. In [LW], among other things, it was shown that the result in $[\mathrm{BW}]$ remain true if (F2), (F3) and (F5) are relaxed to

$\left(\mathrm{F}^{\prime} 2\right) \lim _{|u| \rightarrow \infty} \frac{F(r, u)}{u^{2}}=\infty$;

$\left(\mathrm{F}^{\prime} 5\right) f(r, u) u-2 F(r, u)$ is nondecreasing in $|u|$ and increasing for $|u|>0$ small. One easily checks that by making changes similar to [LW] in our arguments below, Theorem 1.1 remains valid under these changes of assumptions on $f(r, u)$.

The rest of the paper is organized as follows. In section 2, we modify the approach of $[\mathrm{BW}]$ to obtain radially symmetric positive and negative solutions on annuli or balls, by making use of suitable Nehari manifolds. In section 3, we estimate the energy levels of the solutions obtained in section 2 and study the behavior of these energy levels as the underlying domain and $\epsilon$ change, to obtain crucial information which will be needed in section 4 , where the existence and asymptotic profile of the sign changing radial solution are proved. 
2. Positive and negative radial solutions. Setting $v(x)=u(\epsilon x)$, we can reformulate $(1.3)$ as

$$
-\Delta v+b_{\epsilon}(|x|) v=f_{\epsilon}(|x|, v) \text { in } B_{\epsilon},\left.\partial_{\nu} v\right|_{\partial B_{\epsilon}}=0
$$

where $b_{\epsilon}(|x|)=b(\epsilon|x|), f_{\epsilon}(|x|, v)=f(\epsilon|x|, v)$ and $B_{\epsilon}=\left\{x \in \mathbb{R}^{N}:|x|<\epsilon^{-1}\right\}$.

Our arguments in this section are modifications of those in $[\mathrm{BW}]$. We provide sufficient details here for completeness and for the reason that they are needed in the estimates in section 3 later, which play a pivotal role in determining the profile of our sign changing solutions as $\epsilon \rightarrow 0$.

Fix $\epsilon>0$ and $\rho \in\left(0, \epsilon^{-1}\right)$. We consider the mixed boundary value problem

$$
\left\{\begin{array}{c}
-\Delta v+b_{\epsilon}(|x|) v=f_{\epsilon}(|x|, v) \text { for }|x| \in\left(\rho, \epsilon^{-1}\right), \\
v=0 \text { for }|x|=\rho, \partial_{\nu} v=0 \text { for }|x|=\epsilon^{-1} .
\end{array}\right.
$$

In order to obtain a positive solution of (2.2), we consider the modified problem

$$
\left\{\begin{array}{c}
-\Delta v+b_{\epsilon}(|x|) v=g(|x|, v) \text { for }|x| \in\left(\rho, \epsilon^{-1}\right), \\
v=0 \text { for }|x|=\rho, \partial_{\nu} v=0 \text { for }|x|=\epsilon^{-1}
\end{array}\right.
$$

where

$$
g(r, u)=g_{\epsilon}(r, u)= \begin{cases}f_{\epsilon}(r, u) & \text { for } u \geq 0 \\ -f_{\epsilon}(r,-u) & \text { for } u \leq 0\end{cases}
$$

Denote $M=M(\rho)=M\left(\rho, \epsilon^{-1}\right):=\left\{x \in \mathbb{R}^{N}: \rho<|x|<\epsilon^{-1}\right\}$ and let $X=X(M)$ be the Hilbert space of all radial functions in $H^{1}(M)$ satisfying $u(\rho)=0$, with the usual norm

$$
\left[\int_{M}\left(|\nabla u|^{2}+u^{2}\right) d x\right]^{1 / 2}
$$

and $E=E(M)$ be the subspace of $X$ such that

$$
\|u\|^{2}=\|u\|_{M}^{2}:=\int_{M}\left(|\nabla u|^{2}+b_{\epsilon}(|x|) u^{2}\right) d x<\infty .
$$

(In fact, $E=X ; E \neq X$ is possible only when $B_{\epsilon}$ is replaced by $\mathbb{R}^{N}$.) Due to (B), there is a continuous embedding (for $N \geq 3$ )

$$
E \subset X \subset L^{q}(M) \text { for } 2 \leq q \leq \frac{2 N}{N-2},
$$

which is true for $q \in(2, \infty)$ when $N=2$. Define

$$
\phi(u)=\phi_{M}(u)=\phi_{\epsilon, M}(u):=\frac{1}{2} \int_{M}\left(|\nabla u|^{2}+b_{\epsilon}(|x|) u^{2}\right) d x-\int_{M} G(|x|, u) d u,
$$

where $G(r, u)=G_{\epsilon}(r, u)=\int_{0}^{u} g_{\epsilon}(r, v) d v$.

Because of (F1), $\phi$ is well-defined on $E$ and is $C^{1}$, and critical points of $\phi$ are classical solutions of (2.3). A necessary condition for $u \in E$ to be a critical point of $\phi$ is that

$$
\left(\phi^{\prime}(u), u\right):=\frac{1}{2}\|u\|^{2}-\int_{M} g(|x|, u) u d x=0
$$


We define the associated Nehari manifold by

$$
\mathcal{N}=\mathcal{N}_{M}=\mathcal{N}_{\epsilon, M}:=\left\{u \in E \backslash\{0\}:\left(\phi^{\prime}(u), u\right)=0\right\} .
$$

We will show that

$$
\lambda:=\inf _{\mathcal{N}} \phi(u)
$$

is a critical value of $\phi$ with a corresponding positive critical point. To this end, we need several lemmas.

LEMMA 2.1. $\phi$ satisfies the assumptions of the mountain pass theorem on E, namely,

(a) $\phi(0)=0$ and there exist $r_{0}>0, \epsilon_{0}>0$ such that $\phi(u) \geq \epsilon_{0}$ if $\|u\|=r_{0}$.

(b) For every $u \in E \backslash\{0\}$ there exists $t_{0}>0$ such that $\phi(t u)<0$ if $t \geq t_{0}$.

(c) $\phi$ satisfies the Palais-Smale condition.

Proof. This is standard; (a) follows from (B), (F1) and (F4), (b) is a consequence of (F2) and (F3), (c) is implied by (F1) and (F2). We omit the details.

Thus we may apply the mountain pass theorem of Ambrosetti and Rabinowitz to conclude that

$$
c=c_{M}=c_{\epsilon, M}:=\inf _{\gamma \in \Gamma} \max _{t \in[0,1]} \phi(\gamma(t)) \geq \epsilon_{0}>0
$$

is a critical value of $\phi$, where

$$
\Gamma:=\{\gamma \in C([0,1], E): \gamma(0)=0, \phi(\gamma(1))<0\} .
$$

Lemma 2.2. For any $u \in E \backslash\{0\}$, there exists a unique $t=t(u)>0$ such that $t(u) u \in \mathcal{N}$, the maximum of $\phi(t u)$ for $t \in[0, \infty)$ is achieved at $t=t(u)$, and the function from $E \backslash\{0\}$ to $[0, \infty), u \mapsto t(u)$, is continuous.

Proof. Fix $u \in E \backslash\{0\}$ and consider the function

$$
h(t):=\phi(t u), t \in(0, \infty) .
$$

We have

$$
h^{\prime}(t)=\left(\phi^{\prime}(t u), u\right)=t\|u\|^{2}-\int_{M} g(|x|, t u) u d x .
$$

Hence $t u \in \mathcal{N}$ if and only if $h^{\prime}(t)=0$, that is

$$
\|u\|^{2}=\frac{1}{t} \int_{M} g(|x|, t u) u d x .
$$

By (F5), the right-hand side of the above identity is an increasing function of $t$, and by Lemma 2.1, $h(0+0)=0, h(t)>0$ for $t>0$ small and $h(t)<0$ for $t>0$ large. Therefore $\max h(t)$ is achieved at a unique $t=t(u)>0$. It follows that $h^{\prime}(t(u))=0$ and hence $t(u) u \in \mathcal{N}$. The fact that $u \mapsto t(u)$ is continuous follows easily from the uniqueness of $t(u)$ and the boundedness of $t(u)$, the latter follows from (F2), (F3) and (F5), which imply $u g(r, u) \geq a_{2}|u|^{\mu}$ for some $a_{2}>0$ and all $u \in \mathbb{R}^{1}$.

Lemma 2.3. $\lambda=c=d:=\inf _{u \in E \backslash\{0\}} \max _{t \geq 0} \phi(t u)$. 
Proof. By Lemma 2.2 we clearly have $\lambda=d$. Since $\phi(t u)<0$ for $u \in E \backslash\{0\}$ and large $t>0$, we infer $c \leq d$. On the other hand, every $\gamma \in \Gamma$ has to $\operatorname{cross} \mathcal{N}$. This is because, by Lemma 2.2 ,

$$
\mathcal{N}=\{t(u) u: u \in E \backslash\{0\}\}=\partial D,
$$

where

$$
D:=\{t u: u \in E \backslash\{0\}, 0 \leq t<t(u)\},
$$

which contains a neighborhood of 0 in $E$, and $\phi \geq 0$ in $D$. Therefore, $c \geq \lambda$. $\square$

Lemma 2.4. If $u_{0} \in \mathcal{N}$ and $\phi\left(u_{0}\right)=\lambda$, then $u_{0}$ is a critical point of $\phi$.

Proof. This follows from the quantitative deformation lemma, and the proof is exactly the same as in Lemma 3.4 of [BW].

Since $\phi$ satisfies the Palais-Smale condition and $\lambda=c>0, \lambda$ is always achieved by some $u_{0} \in \mathcal{N}$ and hence Lemma 2.4 guarantees that $\phi$ has a critical point $v \in \mathcal{N}$. Since $g(r, u)$ is odd in $u$, we see that $u(x):=|v(x)|$ belongs to $\mathcal{N}$ and $\phi(u)=\phi(v)=\lambda$. Hence $u$ is a nontrivial nonnegative solution of (2.3). Since $f$ is locally Lipschitz in $u$, by the maximum principle, it is positive and hence is a positive solution of (2.2).

We can obtain a negative solution of $(2.2)$ analogously, by defining $g(r, u)$ by

$$
g(r, u)= \begin{cases}-f_{\epsilon}(r,-u) & \text { for } u \geq 0 \\ f_{\epsilon}(r, u) & \text { for } u \leq 0 .\end{cases}
$$

For later reference, we denote the corresponding $\phi, \mathcal{N}, \lambda$, etc. by $\phi^{-}, \mathcal{N}^{-}, \lambda^{-}, \ldots$, respectively.

For fixed $\epsilon>0$ and $0 \leq \rho<\sigma \leq \epsilon^{-1}$, we also consider the Dirichlet problem over $D=D(\rho, \sigma):=$ interior of $\left\{x \in \mathbb{R}^{\bar{N}}: \rho \leq|x|<\sigma\right\}$ :

$$
-\Delta v+b_{\epsilon}(|x|) v=f_{\epsilon}(|x|, v) \text { in } D, v \in H_{0}^{1}(D) .
$$

In this case, we define $X=X(D)$ to be the Hilbert space of all radial functions in $H_{0}^{1}(D)$ with the usual norm, and define $E, \phi, \mathcal{N}, \lambda, c, d$ analogously. The same argument shows that $\phi$ achieves its minimum on $\mathcal{N}$ at a critical point of $\phi$, which gives rise to a positive solution of (2.4). One can also obtain a negative solution of $(2.4)$ by the same trick of redefining $g(r, u)$. To distinguish the solutions for the various situations, we will write $u_{\epsilon, M}^{+}, u_{\epsilon, M}^{-}, u_{\epsilon, D}^{+}, u_{\epsilon, D}^{-}$, with the obvious meanings. Similarly, we will write

$$
\mathcal{N}_{\epsilon, M}^{ \pm}, \lambda_{\epsilon, M}^{ \pm}, \mathcal{N}_{\epsilon, D}^{ \pm}, \lambda_{\epsilon, D}^{ \pm} \text {, etc. }
$$

3. Qualitative properties of $\lambda_{\epsilon, M}^{ \pm}$and $\lambda_{\epsilon, D}^{ \pm}$. In this section, we examine the behavior of $\lambda_{\epsilon, M}^{ \pm}$and $\lambda_{\epsilon, D}^{ \pm}$as $\rho$ and $\sigma$ vary. Since our proof for $\lambda^{+}$and $\lambda^{-}$is the same, we will omit the superscripts + and - and simply write $\lambda_{\epsilon, M}$ or $\lambda_{\epsilon, D}$. To emphasize the dependence on $\rho$ and $\sigma$, we further write

$$
\lambda_{\epsilon, D}=\lambda_{\epsilon, D}(\rho, \sigma), \lambda_{\epsilon, M}=\lambda_{\epsilon, M}(\rho) .
$$

Lemma 3.1. Suppose that (B), (F1)-(F5) are satisfied. Then the following conclusions hold: 
(i) If $0 \leq \rho \leq \rho^{\prime}<\sigma^{\prime} \leq \sigma \leq \epsilon^{-1}$, then $\lambda_{\epsilon, D}(\rho, \sigma) \leq \lambda_{\epsilon, D}\left(\rho^{\prime}, \sigma^{\prime}\right)$.

(ii) If $0<\rho \leq \rho^{\prime}<\epsilon^{-1}$, then $\lambda_{\epsilon, M}(\rho) \leq \lambda_{\epsilon, M}\left(\rho^{\prime}\right) \leq \lambda_{\epsilon, D}\left(\rho^{\prime}, \epsilon^{-1}\right)$.

(iii) For any given $\delta_{0}>0$, there exist positive constants $b_{0}, d_{0}$ and $\sigma_{0}$, all independent of $\epsilon, \rho$ and $\sigma$ such that

$$
\begin{gathered}
\lambda_{\epsilon, D}(\rho, \sigma) \geq b_{0}|D(\rho, \sigma)|^{-\sigma_{0}} \text { for } 0 \leq \rho<\sigma \leq \epsilon^{-1}, \\
\lambda_{\epsilon, M}(\rho) \geq b_{0}\left|M\left(\rho, \epsilon^{-1}\right)\right|^{-\sigma_{0}} \text { for } 0 \leq \rho<\epsilon^{-1} \\
\lambda_{\epsilon, D}(\rho, \sigma) \geq d_{0} \rho^{N-1} \text { whenever } \delta_{0} \leq \rho<\sigma \leq \epsilon^{-1}, \\
\lambda_{\epsilon, M}(\rho) \geq d_{0} \rho^{N-1} \text { whenever } \delta_{0} \leq \rho<\epsilon^{-1}
\end{gathered}
$$

Proof. Since a function in $H_{0}^{1}\left(D\left(\rho^{\prime}, \sigma^{\prime}\right)\right)$ can be regarded as a function in $H_{0}^{1}(D(\rho, \sigma))$ by extension to the value of 0 , we find $\mathcal{N}_{D\left(\rho^{\prime}, \sigma^{\prime}\right)} \subset \mathcal{N}_{D(\rho, \sigma)}$. Hence $\lambda_{\epsilon, D}(\rho, \sigma) \leq \lambda_{\epsilon, D}\left(\rho^{\prime}, \sigma^{\prime}\right)$.

Similarly, $\mathcal{N}_{D\left(\rho^{\prime}, \epsilon^{-1}\right)} \subset \mathcal{N}_{M\left(\rho^{\prime}, \epsilon^{-1}\right)} \subset \mathcal{N}_{M\left(\rho, \epsilon^{-1}\right)}$ implies $\lambda_{\epsilon, M}(\rho) \leq \lambda_{\epsilon, M}\left(\rho^{\prime}\right) \leq$ $\lambda_{\epsilon, D}\left(\rho^{\prime}, \epsilon^{-1}\right)$. This proves (i) and (ii).

By (F2), for every $u \in \mathcal{N}_{D(\rho, \sigma)}$ or $\mathcal{N}_{M\left(\rho, \epsilon^{-1}\right)}$, we have, denoting $\Omega=D(\rho, \sigma)$ or $M\left(\rho, \epsilon^{-1}\right)$,

$$
\phi(u) \geq \frac{1}{2}\|u\|^{2}-\frac{1}{\mu} \int_{\Omega} g(|x|, u) u d x=\left(\frac{1}{2}-\frac{1}{\mu}\right)\|u\|^{2} .
$$

By (F1) and (F4), we can find $a_{3} \geq a_{1}$ such that

$$
\left|g_{\epsilon}(|x|, u)\right| \leq \frac{1}{2} a_{0}|u|+a_{3}|u|^{s} \text { for all } u \in \mathbb{R}^{1}, \epsilon>0 .
$$

Therefore, for $u \in \mathcal{N}_{D(\rho, \sigma)}$ or $\mathcal{N}_{M\left(\rho, \epsilon^{-1}\right)}$ we have

$$
\begin{aligned}
\|u\|^{2} & =\int_{\Omega} g_{\epsilon}(|x|, u) u d x \\
& \leq \frac{1}{2} a_{0} \int_{\Omega} u^{2} d x+a_{3} \int_{\Omega}|u|^{s+1} d x \\
& \leq \frac{1}{2}\|u\|^{2}+a_{3}\left(\int_{\Omega}|u|^{q} d x\right)^{\frac{s+1}{q}}\left(\int_{\Omega} 1 d x\right)^{1-\frac{s+1}{q}} \\
& \leq \frac{1}{2}\|u\|^{2}+a_{3}\|u\|_{L^{q}}^{s+1}|\Omega|^{1-\frac{s+1}{q}}
\end{aligned}
$$

where $s+1<q \leq 2 N /(N-2)$ when $N \geq 3, s+1<q<\infty$ for $N=2$. By the Sobolev embedding theorem, there exists $a_{4}$ depending only on $N$ and $q$ such that $\|u\|_{L^{q}} \leq a_{4}\|u\|$. Hence

$$
\|u\|^{2} \leq \frac{1}{2}\|u\|^{2}+a_{3} a_{4}^{s+1}\|u\|^{s+1}|\Omega|^{1-\frac{s+1}{q}} .
$$

It follows that

$$
\begin{gathered}
\|u\|^{s-1} \geq\left(2 a_{3} a_{4}^{s+1}\right)^{-1}|\Omega|^{\frac{s+1}{q}-1}, \\
\|u\|^{2} \geq a_{5}|\Omega|^{-\sigma_{0}} \text { with } a_{5}=\left(2 a_{3} a_{4}^{s+1}\right)^{\frac{2}{1-s}}, \sigma_{0}=\left(1-\frac{s+1}{q}\right) \frac{2}{s-1} .
\end{gathered}
$$


In view of (3.1), we obtain

$$
\phi(u) \geq b_{0}|\Omega|^{-\sigma_{0}}, b_{0}=\left(\frac{1}{2}-\frac{1}{\mu}\right) a_{5} .
$$

Therefore

$$
\lambda_{\epsilon, M}(\rho) \geq b_{0}\left|M\left(\rho, \epsilon^{-1}\right)\right|^{-\sigma_{0}}, \lambda_{\epsilon, D}(\rho, \sigma) \geq b_{0}|D(\rho, \sigma)|^{-\sigma_{0}} .
$$

To prove the last two inequalities in (iii), we let $\Omega$ denote either $D(\rho, \sigma)$ or $M\left(\rho, \epsilon^{-1}\right)$ and suppose $u=u(r)=u(|x|)$ is a function in $\mathcal{N}_{\Omega}$. Since

$$
\frac{d}{d r}\left(r^{N-1} u^{2}\right)=2 u u_{r} r^{N-1}+(N-1) u^{2} r^{N-2}
$$

and $u(\rho)=0$, we have, for $r>\rho \geq \delta_{0}$,

$$
\begin{aligned}
r^{N-1} u^{2}(r) & =2 \int_{\rho}^{r} u u_{r} s^{N-1} d s+(N-1) \int_{\rho}^{r} u^{2} s^{N-2} d s \\
& \leq 2 \int_{\Omega} u|\nabla u| d x+(N-1) \rho^{-1} \int_{\Omega} u^{2} d x \\
& \leq 2\|u\|_{L^{2}}\|\nabla u\|_{L^{2}}+(N-1) \delta_{0}^{-1}\|u\|_{L^{2}}^{2} .
\end{aligned}
$$

It follows that

$$
u(r) \leq r^{(1-N) / 2}\left(2\|u\|_{L^{2}}\|\nabla u\|_{L^{2}}+(N-1) \delta_{0}^{-1}\|u\|_{L^{2}}^{2}\right)^{1 / 2} \leq a_{6} \rho^{(1-N) / 2}\|u\| .
$$

Hence

$$
\begin{aligned}
\|u\|^{2} & \leq \frac{1}{2} a_{0}\|u\|_{L^{2}}^{2}+a_{3} \int_{\Omega}|u|^{s+1} d x \\
& \leq \frac{1}{2}\|u\|^{2}+a_{3} \int_{\Omega} u^{2}\left(a_{6} \rho^{(1-N) / 2}\|u\|\right)^{s-1} d x \\
& =\frac{1}{2}\|u\|^{2}+a_{3} a_{6}^{s-1} \rho^{\frac{1-N}{2}(s-1)}\|u\|^{s-1}\|u\|_{L^{2}}^{2} \\
& \leq \frac{1}{2}\|u\|^{2}+a_{7}\|u\|^{s+1} \rho^{\frac{1-N}{2}(s-1)} .
\end{aligned}
$$

We thus deduce

$$
\|u\|^{s-1} \geq\left(2 a_{7}\right)^{-1} \rho^{\frac{N-1}{2}(s-1)},
$$

and

$$
\phi(u) \geq\left(\frac{1}{2}-\frac{1}{\mu}\right)\|u\|^{2} \geq\left(\frac{1}{2}-\frac{1}{\mu}\right)\left(2 a_{7}\right)^{\frac{2}{1-s}} \rho^{N-1} .
$$

The last two inequalities in (iii) hence follows.

Lemma 3.2. Suppose that $0 \leq \rho<\sigma<\epsilon_{0}^{-1}$. Then there exists $C_{0}>0$ depending on $D(\rho, \sigma)$ but independent of $\epsilon \in\left(0, \epsilon_{0}\right]$, and $c_{0}>0$ independent of $\rho, \sigma, \epsilon$ such that

$$
c_{0} \leq \lambda_{\epsilon, D}(\rho, \sigma) \leq C_{0}, \lambda_{\epsilon, M}(\rho) \leq C_{0} .
$$


Proof. As before, by (F2), (F3) and (F5) there exists $a_{2}>0$ independent of $\epsilon$ such that

$$
G_{\epsilon}(r, u) \geq a_{2}|u|^{\mu} \text { for } u \in \mathbb{R}^{1}, \epsilon>0 .
$$

Therefore, if we fix a continuous function $u_{0} \in E \backslash\{0\}$, then

$$
\begin{aligned}
\phi\left(t u_{0}\right) & =\frac{t^{2}}{2}\left\|u_{0}\right\|^{2}-\int_{D(\rho, \sigma)} G_{\epsilon}\left(|x|, t u_{0}\right) d x \\
& \leq \frac{t^{2}}{2}\left\|u_{0}\right\|^{2}-a_{2} t^{\mu} \int_{D(\rho, \sigma)}\left|u_{0}\right|^{\mu} d x \\
& \leq 0
\end{aligned}
$$

for $t$ satisfying

$$
t \geq t_{0}:=\left(\frac{\left\|u_{0}\right\|^{2}}{2 a_{2}} / \int_{D(\rho, \sigma)}\left|u_{0}\right|^{\mu} d x\right)^{1 /(\mu-2)} .
$$

By Lemma 2.3 and the definition of $d$, we obtain

$$
\begin{aligned}
\lambda_{\epsilon, D}(\rho, \sigma) & \leq \max _{t \in\left[0, t_{0}\right]} \phi\left(t u_{0}\right) \\
& \leq \max _{t \in\left[0, t_{0}\right]} \frac{t^{2}}{2}\left\|u_{0}\right\|^{2} \\
& =C_{0}:=\frac{t_{0}^{2}}{2}\left\|u_{0}\right\|^{2} .
\end{aligned}
$$

By Lemma 3.1,

$$
\lambda_{\epsilon, M}(\rho) \leq \lambda_{\epsilon, D}\left(\rho, \epsilon^{-1}\right) \leq \lambda_{\epsilon, D}(\rho, \sigma) \leq C_{0} .
$$

On the other hand, by (F1), (F4) and the Sobolev embedding theorem, there exists $\eta_{0}>0$ independent of $D(\rho, \sigma)$ and $\epsilon$ such that

$$
\phi(u) \geq \frac{1}{3}\|u\|^{2} \text { if } u \in E \text { satisfies }\|u\| \leq \eta_{0} .
$$

It then follows from the mountain pass characterization of $c=c_{D(\rho, \sigma)}$ that

$$
\lambda_{\epsilon, D}(\rho, \sigma) \geq c_{0}:=\eta_{0}^{2} / 3
$$

Lemma 3.3. If $\rho_{n} \rightarrow \rho \in\left(0, \epsilon^{-1}\right)$ and $\sigma_{n} \rightarrow \sigma \in\left(\rho, \epsilon^{-1}\right)$ as $n \rightarrow \infty$, then

$$
\begin{aligned}
\lambda_{\epsilon, D}(0, \sigma) & \leq \underline{\lim }_{n \rightarrow \infty} \lambda_{\epsilon, D}\left(0, \sigma_{n}\right), \\
\lambda_{\epsilon, D}(\rho, \sigma) & \leq \underline{\lim }_{n \rightarrow \infty} \lambda_{\epsilon, D}\left(\rho_{n}, \sigma_{n}\right), \\
\lambda_{\epsilon, M}(\rho) & \leq \underline{\lim }_{n \rightarrow \infty} \lambda_{\epsilon, M}\left(\rho_{n}\right) .
\end{aligned}
$$

Proof. This follows from a simple modification of the proof of Proposition 4.1 part d) in $[\mathrm{BW}]$. We omit the details. $\square$ 
4. Existence and asymptotic profile. Set

$$
s_{j}= \begin{cases}+ & \text { for } j \text { even }, \\ - & \text { for } j \text { odd }\end{cases}
$$

and define, similar to $[\mathrm{BW}]$,

$$
\Lambda_{\epsilon}^{+}\left(\rho_{1}, \cdots, \rho_{k}\right)=\sum_{j=0}^{k-1} \lambda_{\epsilon, D}^{s_{j}}\left(\rho_{j}, \rho_{j+1}\right)+\lambda_{\epsilon, M}^{s_{k}}\left(\rho_{k}\right),
$$

for $0=\rho_{0}<\rho_{1}<\ldots<\rho_{k}<\epsilon^{-1}$. For fixed $\epsilon>0$, it follows from Lemmas 3.1 and 3.3 that $\Lambda_{\epsilon}^{+}$attains its minimum at some point $\left(\rho_{1}^{\epsilon}, \ldots, \rho_{k}^{\epsilon}\right)$, and corresponding to each $\lambda_{\epsilon, D}^{+}\left(\rho_{j}^{\epsilon}, \rho_{j+1}^{\epsilon}\right)$ with $j$ even, by our results in section 2 , there is a positive radial solution $u_{\epsilon, j}^{+}$of (2.4) with $D=D\left(\rho_{j}^{\epsilon}, \rho_{j+1}^{\epsilon}\right)$, and corresponding to each $\lambda_{\epsilon, D}^{-}\left(\rho_{j}^{\epsilon}, \rho_{j+1}^{\epsilon}\right)$ with $j$ odd, there is a negative radial solution $u_{\epsilon, j}^{-}$of (2.4) with $D=D\left(\rho_{j}^{\epsilon}, \rho_{j+1}^{\epsilon}\right)$. Moreover, we have

$$
u_{\epsilon, j}^{s_{j}} \in \mathcal{N}_{D\left(\rho_{j}^{\epsilon}, \rho_{j+1}^{\epsilon}\right)}, \phi_{D\left(\rho_{j}^{\epsilon}, \rho_{j+1}^{\epsilon}\right)}^{s_{j}}\left(u_{\epsilon, j}^{s_{j}}\right)=\lambda_{\epsilon, D}^{s_{j}}\left(\rho_{j}^{\epsilon}, \rho_{j+1}^{\epsilon}\right) .
$$

Similarly, $\lambda_{\epsilon, M}^{s_{k}}\left(\rho_{k}^{\epsilon}\right)$ corresponds to a radial solution $u_{\epsilon, k}^{s_{k}}$ of $(2.2)$, whose sign is the same as $s_{k}$, and

$$
u_{\epsilon, k}^{s_{k}} \in \mathcal{N}_{M\left(\rho_{k}^{\epsilon}, \epsilon^{-1}\right)}, \phi_{M\left(\rho_{k}^{\epsilon}, \epsilon^{-1}\right)}^{s_{k}}\left(u_{\epsilon, k}^{s_{k}}\right)=\lambda_{\epsilon, M}^{s_{k}}\left(\rho_{k}^{\epsilon}\right) .
$$

We now define $u_{\epsilon}^{+} \in H^{1}\left(B_{\epsilon}\right)$ by

$$
u_{\epsilon}^{+}(x)=u_{\epsilon, j}^{s_{j}}(x) \text { if } \rho_{j}^{\epsilon} \leq|x|<\rho_{j+1}^{\epsilon}, j=0, \ldots, k, \rho_{0}^{\epsilon}=0, \rho_{k+1}^{\epsilon}=\epsilon^{-1} .
$$

By exactly the same argument used in the proof of Lemma 5.1 in [BW], we conclude that $u_{\epsilon}^{+}$is a solution of $(2.1)$.

If we define

$$
s_{j}= \begin{cases}- & \text { for } j \text { even }, \\ + & \text { for } j \text { odd },\end{cases}
$$

then the above analysis gives rise to a radial solution $u_{\epsilon}^{-}(|x|)$ of (2.1) which satisfies $u_{\epsilon}^{-}(0)<0$ with exactly $k$ zeros in $\left(0, \epsilon^{-1}\right)$.

We next study the asymptotic profile of $u_{\epsilon}^{+}$as $\epsilon \rightarrow 0$; the analysis for $u_{\epsilon}^{-}$is similar. To simplify notations, we write $u_{\epsilon}$ instead of $u_{\epsilon}^{+}$.

LEMma 4.1. Fix $\epsilon_{0}>0$ small, let $\epsilon \in\left(0, \epsilon_{0}\right]$ and $u_{\epsilon}$ be the radial solution of (2.2) described above, and $0<\rho_{1}^{\epsilon}<\ldots<\rho_{k}^{\epsilon}<\epsilon^{-1}$ the zeros of $u_{\epsilon}(r)$. Then there exists $\delta>0$ independent of $\epsilon$ such that

$\rho_{j+1}^{\epsilon}-\rho_{j}^{\epsilon} \geq \delta, \quad \rho_{k}^{\epsilon} \leq \delta^{-1}$ for $\epsilon \in\left(0, \epsilon_{0}\right], j=0, \ldots, k, \rho_{0}^{\epsilon}=0, \rho_{k+1}^{\epsilon}=\epsilon^{-1}$.

Proof. Fix $0<\rho_{1}^{*}<\ldots<\rho_{k}^{*}<\epsilon_{0}^{-1}$. By Lemma 3.2, we obtain

$$
\begin{aligned}
\Lambda_{\epsilon}\left(\rho_{1}^{\epsilon}, \ldots, \rho_{k}^{\epsilon}\right) & \leq \Lambda_{\epsilon}\left(\rho_{1}^{*}, \ldots, \rho_{k}^{*}\right) \\
& =\sum_{j=0}^{k-1} \lambda_{\epsilon, D}^{s_{j}}\left(\rho_{j}^{*}, \rho_{j+1}^{*}\right)+\lambda_{\epsilon, M}^{s_{k}}\left(\rho_{k}^{*}\right) \\
& \leq(k+1) C_{0}
\end{aligned}
$$


for all $\epsilon \in\left(0, \epsilon_{0}\right]$, where $C_{0}$ depends on $\left(\rho_{1}^{*}, \ldots, \rho_{k}^{*}\right)$ but is independent of $\epsilon$. In view of Lemma 3.1, we necessarily have

$$
\rho_{j+1}^{\epsilon}-\rho_{j}^{\epsilon} \geq \delta, \rho_{k}^{\epsilon} \leq \delta^{-1}
$$

for some $\delta>0$ independent of $\epsilon$.

THEOREM 4.2. For any positive sequence $\epsilon_{n} \rightarrow 0$, there exists a subsequence $\left\{\epsilon_{n_{i}}\right\}$ such that along this subsequence $u_{\epsilon} \rightarrow U$ in $C_{\text {loc }}^{1}\left(\mathbb{R}^{N}\right)$, where $U=U(r)$ is a radial solution of (1.4) satisfying $U(0)>0$, with exactly $k$ zeros: $0<\rho_{1}<\ldots<\rho_{k}<\infty$. Moreover, $\rho_{j}^{\epsilon} \rightarrow \rho_{j}$ along $\epsilon_{n_{i}}$ for $j=1, \ldots, k$.

Proof. To simplify notations we will write $u_{n}=u_{\epsilon_{n}}$. Using (4.1), (4.2), (4.4) and (3.1), we find that $\left\{\left\|u_{\epsilon}\right\|_{H^{1}\left(B_{\epsilon}\right)}: 0<\epsilon \leq \epsilon_{0}\right\}$ is bounded. Since by (4.3) we have $\rho_{1}^{\epsilon} \geq \delta$, we may now apply (3.2) to conclude that

$$
u_{\epsilon}(r) \leq C r^{(1-N) / 2} \text { for all } r \geq \rho_{1}^{\epsilon} \text { and some } C>0 \text { independent of } \epsilon .
$$

Using the boundedness of $\left\{\left\|u_{n}\right\|_{H^{1}\left(B_{\epsilon_{n}}\right)}\right\}$, (F1) and a standard bootstrapping argument, we find that

$$
u_{n}(r) \leq C \text { for } 0 \leq r \leq \rho_{1}^{\epsilon_{n}}, n \geq 1 .
$$

Therefore $\left\{\left\|u_{n}\right\|_{L^{\infty}\left(B_{\epsilon_{n}}\right)}\right\}$ is bounded, and hence

$$
\left|f_{\epsilon_{n}}\left(|x|, u_{n}\right)\right| \leq C
$$

for some $C>0$ and all $n \geq 1$ and $x \in B_{\epsilon_{n}}$. We may now apply standard elliptic regularity theory (see $[\mathrm{GT}]$ ) to the equation satisfied by $u_{n}$, and a diagonal process to extract a subsequence $\left\{u_{n_{i}}\right\}$ of $\left\{u_{n}\right\}$ such that $u_{n_{i}} \rightarrow U$ in $C_{l o c}^{1}\left(\mathbb{R}^{N}\right)$. It is easily seen that $U$ solves the differential equation in (1.4) in the weak sense. Since each $u_{n}$ is radial, so is $U$. Since $\left\{\left\|u_{n}\right\|_{H^{1}\left(B_{\epsilon_{n}}\right)}\right\}$ is bounded, say $\left\|u_{n}\right\|_{H^{1}\left(B_{\epsilon_{n}}\right)} \leq C$, we deduce that $\|U\|_{H^{1}\left(B_{\epsilon}\right)} \leq C$ for each $\epsilon>0$. It follows that $U \in H^{1}\left(\mathbb{R}^{N}\right)$, and by bootstrapping it is a classical solution of (1.4). We may assume that $\rho_{1}^{\epsilon_{n_{i}}} \rightarrow \rho_{1} \in\left[\delta, \delta^{-1}\right]$. Then by Lemma 3.2 we deduce that

$$
\phi_{0, D\left(0, \rho_{1}\right)}(U)=\lim _{i \rightarrow \infty} \phi_{\epsilon_{n_{i}}, \rho_{1} \epsilon_{i}}\left(u_{n_{i}}\right) \geq c_{0}>0 .
$$

This implies that $U \not \equiv 0$. Since $f$ is locally Lipschitz in $u$, by the uniqueness of the initial value problem for ODEs (we remark that this is not affected by the singularity of the ODE for $U(r)$ at $r=0$ ), necessarily $U(0)>0$ and $U$ has exactly $k$ nondegenerate zeros $0<\rho_{1}<\ldots<\rho_{k} \leq \delta^{-1}$, for otherwise, for large $i, u_{n_{i}}$ would not have exactly $k$ zeros with none of which lying outside $\left(0, \delta^{-1}\right]$. It then follows that $\rho_{j}^{\epsilon_{n_{i}}} \rightarrow \rho_{j}$ as $i \rightarrow \infty$, for every $j=1, \ldots, k$. $\square$

It is easily seen that Theorem 1.1 follows from (4.3), (4.5) and Theorem 4.2.

REMARK 4.3. If we replace the Neumann boundary condition in (1.3) by the homogeneous Dirichlet boundary condition, then our analysis carries over with no extra difficulties. Hence the conclusions in Theorem 1.1 remain valid for this case. Our arguments can also be easily modified to treat the case where we replace $B$ by $\mathbb{R}^{N}$ and require $u \in H^{1}\left(\mathbb{R}^{N}\right)$ in (1.3). Therefore the conclusions of Theorem 1.1 hold for this case as well. Moreover, Remark 1.2 also applies to these cases. 
Acknowledgements. This work was initiated during the visits of $\mathrm{Y}$. Du to University of Rome I (Italy) and Capital Normal University (China). He gratefully acknowledges the warm hospitalities received during the visits.

\section{REFERENCES}

[AMn1] A. Ambrosetti, A. Malchiodi And W.M. Ni, Singularly perturbed elliptic equations with symmetry: existence of solutions concentrating on spheres. I, Comm. Math. Phys., 235 (2003), pp. 427-466.

[Amn2] A. Ambrosetti, A. Malchiodi And W.M. Ni, Singularly perturbed elliptic equations with symmetry: existence of solutions concentrating on spheres. II, Indiana Univ. Math. J., 53 (2004), pp. 297-329.

[BCW $]$ T. Bartsch, M. Clapp And T. Weth, Configuration spaces, transfer, and 2-nodal solutions of a semiclassical nonlinear Schrödinger equation, Math. Ann., 338 (2007), pp. $147-185$.

[BW] T. BARTSCH AND M. WILLEM, Infinitely many radial solutions of a semilinear elliptic problem on $\mathbb{R}^{N}$, Arch. Rational Mech. Anal., 124 (1993), pp. 261-276.

[CDNY] D. CAO, E.N. Dancer, E.S. Noussair AND S. Yan, On the existence and profile of multi-peaked solutions to singularly perturbed semilinear Dirichlet problems, Discrete Contin. Dynam. Systems, 2 (1996), pp. 221-236.

[CZ] D. CAO AND X. ZHU, On the existence and nodal character of solutions of semilinear elliptic equations, Acta Math. Sci. (English Ed.), 8 (1988), pp. 345-359.

[CSS] G. Cerami, S. Solimini and M. Struwe, Some existence results for superlinear elliptic boundary value problem involving critical exponents, J. Funct. Anal., 69 (1986), pp. 289-306.

[DP] T. D'APrile AND A. Pistoia, On the number of sign-changing solutions of a semiclassical nonlinear Schrödinger equation, Adv. Differential Equations, 12 (2007), pp. 737-758.

[GT] D. Gilbarg and N.S. Trudinger, Elliptic Partial Differential Equations of Second Order, Berlin, New York: Springer 1983.

[GW] C. GUI AND J. WeI, On multiple mixed interior and boundary peak solutions for some singularly perturbed Neumann problems, Can. J. Math., 52 (2000), pp. 522-538.

[LW] Z. LiU AND Z.Q. WANG, On the Ambrosetti-Rabinowitz superlinear condition, Adv. Nonlinear Stud., 4 (2004), pp. 563-574.

[MM] A. Malchiodi and M. Montenegro, Multidimensional boundary layers for a singularly perturbed Neumann problem, Duke Math. J., 124 (2004), pp. 105-143.

[MNW] A. Malchiodi, W.M. Ni AND J. WeI, Multiple clustered layer solutions for semilinear Neumann problems on a ball, Ann. I. H. Poincare Anal. Non Lineaire, 22 (2005), pp. 141-163.

[N] W.M. Ni, Qaulitative properties of solutions to elliptic problems, Handbook of Diff. Eqns., Stationary Partial Differential Equations, Vol. 1, Ed. M Chipot and P. Quittner, Elsevier, 2004, pp.157-233. 